\title{
RF Magnetic Shielding Effects of a Bincho-Charcoal Plate
}

\author{
Keisuke Itoh* $^{*}$ Member \\ Mineo Itoh* Member
}

\begin{abstract}
Recently, there has been increased interest for electromagnetic shielding in the radio frequency (RF) region. The use of effective electromagnetic shields has, moreover, been required to improve the electromagnetic environment. The present paper has applied bincho-charcoal, a high quality charcoal found in Japan, to improve RF electromagnetic shields. Bincho-charcoal makes an excellent shield, due to a very large value of relative permittivity in the RF region. In the present research, the evaluation of the RF magnetic shielding degree $S D_{\mathrm{H}}$ of the bincho-charcoal was limited to a plate being exposed to an electromagnetic wave. The $S D_{\mathrm{H}}$ of the single plate was found to increase with an increase in RF frequency over the range from 1 $\mathrm{MHz}$ to $1 \mathrm{GHz}$. The value of $S D_{\mathrm{H}}$ at $100 \mathrm{MHz}$ was found to be about $15 \mathrm{~dB}$. The present authors have, furthermore, improved the value of $S D_{\mathrm{H}}$ by the use of a triple plate, constructed from three bincho-charcoal plates. The $S D_{\mathrm{H}}$ of the triple plate at $100 \mathrm{MHz}$ was found to be improved by about $15 \mathrm{~dB}$ over that of the single plate. In addition, the $S D_{\mathrm{H}}$ was shown to be improved by the superposition of a copper plate over the triple plate.
\end{abstract}

Keywords : bincho-charcoal plate, relative permittivity, RF magnetic shielding effect, RF electric shielding effect.

\section{Introduction}

Recently, electromagnetic environmental problems have become more serious ${ }^{(1)}$. In general, therefore, there has been a rising need for electromagnetic shields in the radio frequency (RF) region. As one of the basic areas of research for the improvement of the electromagnetic environment, the present paper has applied bincho-charcoal, a high quality charcoal found in Japan, as RF magnetic shields ${ }^{(2)}$. This is advantageous since the relative permittivity of bincho-charcoals has a very large value $\left(10^{7}-10^{2}\right)$ in the $\mathrm{RF}$ region $(1 \mathrm{MHz}-1 \mathrm{GHz})$.

In the present research, the evaluation of the RF magnetic shielding degree $S D_{\mathrm{H}}$ for the bincho-charcoal is limited to a plate (100 $\mathrm{mm}$ square, $5 \mathrm{~mm}$ thick) being exposed to an electromagnetic wave. It was found that the value of $S D_{\mathrm{H}}$ for a single plate in the frequency region of $1 \mathrm{MHz}$ to $1 \mathrm{GHz}$ increased with frequency, and had a value of $15 \mathrm{~dB}$ at $100 \mathrm{MHz}$. The present authors have, furthermore, improved the value of $S D_{\mathrm{H}}$ by the use of double and triple plates, constructed from two or three bincho-charcoal plates. The $S D_{\mathrm{H}}$ of triple plate at $100 \mathrm{MHz}$ was found to be improved by about $15 \mathrm{~dB}$ over that of the single plate.

Experimental results revealed several characteristics of the bincho-charcoal plate that include the dependence of the relative permittivity of the bincho-charcoal on radio frequency $(1 \mathrm{MHz}-1$ $\mathrm{GHz}$ ), the dependence of $S D_{\mathrm{H}}$ on radio frequency, the nondependence of $S D_{\mathrm{H}}$ on the value of RF output power, the nondependence of $S D_{\mathrm{H}}$ on the value of thickness, and the dependence of $S D_{\mathrm{H}}$ on the value of surface area. Also included are the characteristics of the RF electric shielding effect $S D_{\mathrm{E}}$ for the bincho-charcoal plates as a function of radio frequency. In addition, the RF magnetic shielding effect revealed to be improved by the superposition of a copper plate over the triple bincho-charcoal plate.

\footnotetext{
Interdisci. Grad. School of Science and Engineering, Kinki University

3-4-1 Kowakae, Higashi-Osaka, Osaka 577-8502
}

\section{Experimental Procedure}

2.1 Fabrication of the Bincho-charcoal Plate The bincho-charcoal, as the absorbing material for the electromagnetic wave, is obtained from very hard oak, sintered under high temperature conditions $\left(1000{ }^{\circ} \mathrm{C}\right.$ for example) and then rapidly cooled. This charcoal has the properties hardness, fewer fissures, and less warping compared with other charcoals, and is well-known in Japan as a high quality charcoal.

The bincho-charcoal tiles (10 $\mathrm{mm}$ square, $5 \mathrm{~mm}$ thick) were fabricated by use of a diamond saw at low cutting rates to inhibit the effect of heat. The paste for gluing the tiles was prepared by homogeneous mixing bincho-charcoal powder (about $2 \mu \mathrm{m}$ or smaller) with polyvinyl-alcohol. And the weight ratio of the powder to polyvinyl-alcohol was $1: 1$. The tiles were glued using the paste and then dried naturally in the room temperature $(300 \mathrm{~K})$ for about 8 hours. Figure 1 shows a part of the geometry of a typical bincho-charcoal plate (100 $\mathrm{mm}$ square, $5 \mathrm{~mm}$ thick).

Typical surface scanning electron microscope (SEM) photograph of the bincho-charcoal tile is shown in Fig. 2. The bincho-charcoal tile exhibits the scanty porous structure without cracks.

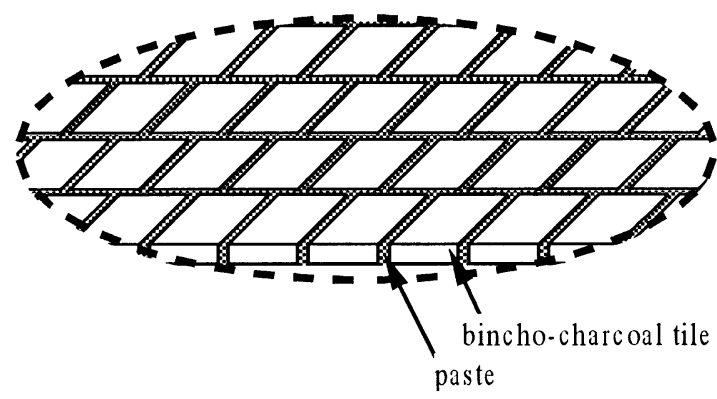

Fig. 1. Sketch of part of the geometry of a typical bincho-charcoal plate. 


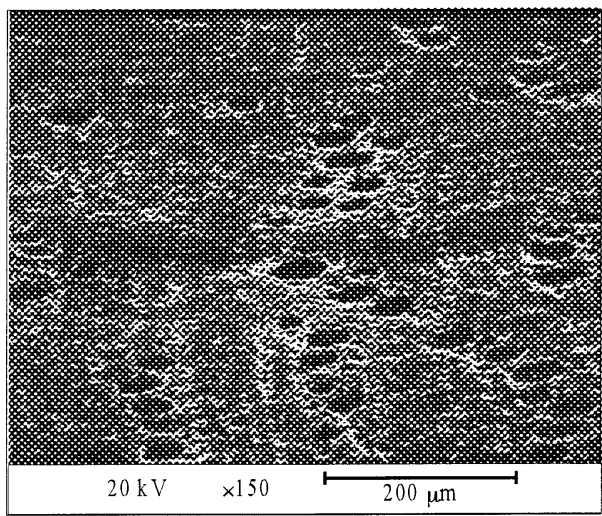

Fig. 2. Typical surface SEM microphotograph of a bincho-charcoal tile.

2.2 Measuring system for the RF Electromagnetic Shielding Effect Figure 3 is a schematic diagram illustrating the experimental arrangement used to measure the RF electromagnetic shielding effects, $S D_{\mathrm{H}}$ and $S D_{\mathrm{E}}$, at the center of a bincho-charcoal plate under temperature condition of $300 \mathrm{~K}^{(2) \sim(5)}$. In the present research, it is measured the $S D_{\mathrm{H}}$ and $S D_{\mathrm{E}}$ under the constant air space $\left(3.4 \mathrm{~m}^{3}\right)$. The RF output, in the frequency region from $1 \mathrm{MHz}$ to $1 \mathrm{GHz}$, of the tracking generator (HP 8594) having a spectrum analyzer, is amplified by $40 \mathrm{~dB}$ by use of a broad band power amplifier (EIN 411LA). The amplified output is then guided to a transmitting antenna. The signal from the receiving antenna is amplified by $71 \mathrm{~dB}$ by use of two preamplifiers (HP 847D and NF SA-230F5), and guided to the input terminal of the spectrum analyzer. The results from the spectrum analyzer are then transferred through GPIB to a lap-top computer.

In the RF shielding system, propagation of the electromagnetic wave from the transmitting antenna to receiving antenna was always arrayed to be perpendicular to the surface of the shielding plate. In this measuring system, the coaxial cable employed as the receiving line was threaded through ferrite rings, in order to reduce any mutual relationship between the transmitting and receiving lines, such as reported in Ref. (2).

The electromagnetic shielding degree $S D$ can be specified in terms of the reduction in magnetic and/or electric field strength due to the shielding material ${ }^{(6)}$. In general, $S D_{\mathrm{H}}$ is defined as $S D_{\mathrm{H}}=10 \log \left(P_{\mathrm{H} 0} / P_{\mathrm{H} 1}\right)$. Here, $P_{\mathrm{H} 0}$ and $P_{\mathrm{H} 1}$ are the strengths of the incident magnetic field power and the magnetic field of the transmitted wave as it emerges from the bincho-charcoal plate, respectively. Also, the electric shielding degree $S D_{\mathrm{E}}$ is defined as $S D_{\mathrm{E}}=10 \log \left(P_{\mathrm{E} 0} / P_{\mathrm{E} 1}\right)$, where $P_{\mathrm{E} 0}$ and $P_{\mathrm{E} 1}$ are the output power of the electric field power of the receiving antenna without and with the bincho-charcoal plate, respectively. The input power of the transmitting antenna and the distance $(30.0 \mathrm{~mm})$ between the transmitting and receiving antennas are held constant. Loop and probe antennas are used for the measurements of the magnetic shielding degree $S D_{\mathrm{H}}$ and electric shielding degree $S D_{\mathrm{E}}$, respectively.

\section{Results and Discussion}

3.1 Relative Permittivity In general, the relative permittivity $\varepsilon_{\mathrm{r}}$ for a dielectric, in the radio frequency RF region, can be expressed as $\varepsilon_{\mathrm{r}}=\varepsilon_{\mathrm{r}}{ }^{\prime}-j \varepsilon_{\mathrm{r}}$ ", where $\varepsilon_{\mathrm{r}}$ ' and $\varepsilon_{\mathrm{r}}$ " are the real and imaginary relative permittivities, respectively and $j$ is the

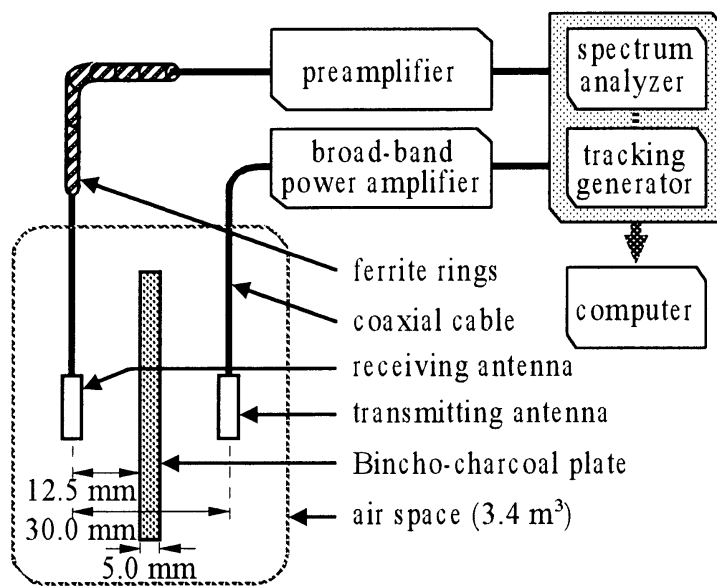

Fig. 3. Schematic diagram of the experimental arrangement used to measure the electromagnetic shielding characteristics.

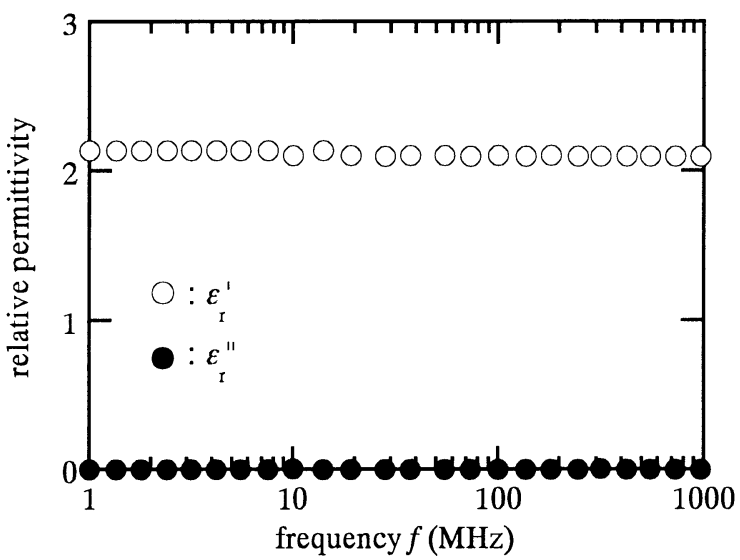

(a) Teflon

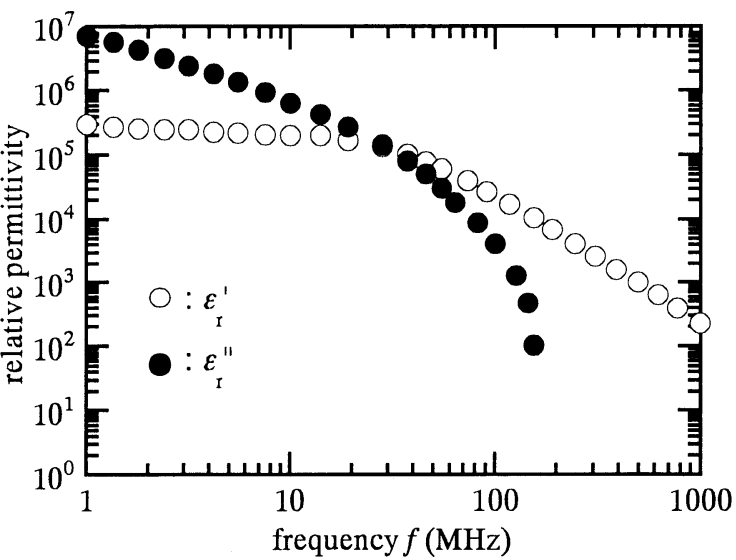

(b) Bincho-charcoal

Fig. 4. Typical characteristics of the relative permittivity of (a) teflon and (b) bincho-charcoal as functions of frequency $f$. The open and solid circles represent the real $\varepsilon_{\mathrm{r}}$ ' and imaginary $\varepsilon_{\mathrm{r}}$ " relative permittivities, respectively.

imaginary unit. In addition, the values of $\varepsilon_{\mathrm{r}}$ form the determinant for the electromagnetic shielding effects.

Figure 4 (a) shows the dependence of the values of $\varepsilon_{\mathrm{r}}$ ' (open circles) and $\varepsilon_{\mathrm{r}}$ " (solid circles) for a teflon shield (as the standard material for measuring the relative permittivity in the RF region), 
on the frequency $f$ in the frequency region from $1 \mathrm{MHz}$ to $1 \mathrm{GHz}$. The values of $\varepsilon_{\mathrm{r}}$ ' and $\varepsilon_{\mathrm{r}}$ " remain constant versus the values of $f$, and reflect very low values compared with those of binchocharcoal.

Figure 4 (b) shows the typical values of $\varepsilon_{\mathrm{r}}$ ' (open circles) and $\varepsilon_{\mathrm{r}}$ " (solid circles) for the bincho-charcoal shield, as functions of frequency $f$, over the radio frequency range from $1 \mathrm{MHz}$ to $1 \mathrm{GHz}$. It can be seen that the characteristics of $\varepsilon_{\mathrm{r}}\left(\varepsilon_{\mathrm{r}}, \varepsilon_{\mathrm{r}}^{\prime}\right)$ decrease as the frequency increases. The values of $\varepsilon_{\mathrm{r}}\left(\varepsilon_{\mathrm{r}}^{\prime}, \varepsilon_{\mathrm{r}}^{\prime \prime}\right)$ for the binchocharcoal are very large $\left(10^{7}-10^{2}\right)$ in the RF region. Therefore, bincho-charcoal can be considered as the material for use as electromagnetic shields. These values were measured by use of a RF impedance material analyzer (HP 4291A).

3.2 RF Magnetic Shielding Effect of Bincho-charcoal Plate Figure 5 shows the RF magnetic shielding degree $S D_{\mathrm{H}}$ of the bincho-charcoal plates as a function of frequency $f$, over the frequency range from $1 \mathrm{MHz}$ to $1 \mathrm{GHz}$, under a constant $\mathrm{RF}$ output power $P_{\mathrm{H}}(33 \mathrm{dBm})$ of the transmitting antenna. The value of the power flux density at the center of bincho-charcoal plate is about $10 \mathrm{~W} / \mathrm{m}^{2}$ at $100 \mathrm{MHz}$. In this figure, the curves formed by the open squares, solid circles, and open triangles represent the shielding characteristics for the single, double, and triple plates of bincho-charcoal, respectively. As can be seen in Fig. 5, the values of $S D_{\mathrm{H}}$ for the plates increase as the values of the frequency $f$ increase, in the frequency region from $1 \mathrm{MHz}$ to $1 \mathrm{GHz}$. It can also be seen that the value of $S D_{\mathrm{H}}$ for the triple plate at $100 \mathrm{MHz}$ was improved by about $15 \mathrm{~dB}$ over that of the single plate. That is, the value of $S D_{\mathrm{H}}$ was remarkably improved as a function of the superposition-layer number of the bincho-charcoal plate.

In addition, the values of $S D_{\mathrm{H}}$ were systematically measured by changing the air gap distance $\delta$ between the two bincho-charcoal plates in the double plate shield. The dependence of $S D_{\mathrm{H}}$ for $\delta$ of $10 \mathrm{~mm}$ on the $f$ exhibited similar characteristics such as those shown for the triple plate (open triangles) in Fig. 5. Namely, the value of $S D_{\mathrm{H}}$ for $\delta$ of $10 \mathrm{~mm}$ for the double plate at $100 \mathrm{MHz}$ was improved by about $13 \mathrm{~dB}$ over that of the single plate.

The characteristics of $S D_{\mathrm{H}}$ for values of RF output power $P_{\mathrm{H}}$ of the transmitting antenna for the bincho-charcoal plates at $1 \mathrm{GHz}$ are displayed in Fig. 6. The open squares, solid circles, and open

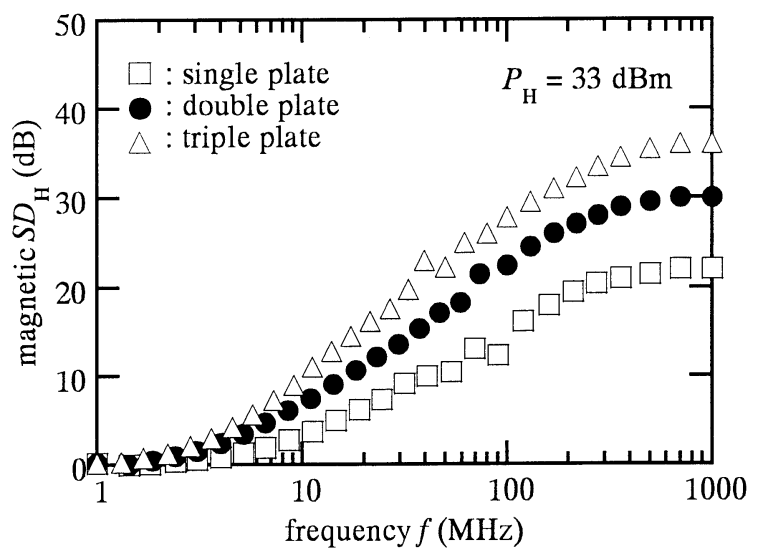

Fig. 5. RF magnetic shielding degree $S D_{\mathrm{H}}$ for the binchocharcoal plates as functions of radio frequency $f$. The open triangles, solid circles, and open squares represent the values of $S D_{\mathrm{H}}$ for the triple plate, double plate, and single plate of bincho-charcoal, respectively, under a constant RF output power $P_{\mathrm{H}}(33 \mathrm{dBm})$ of the transmitting antenna. triangles represent the single, double, and triple plates of the charcoal, respectively. It can be seen that the characteristics of $S D_{\mathrm{H}}$ for the bincho-charcoal plates display no evidence of dependence on the values of $P_{\mathrm{H}}$ in the region between $3 \mathrm{dBm}$ and $33 \mathrm{dBm}$. Namely, the values of $S D_{\mathrm{H}}$ remain constant and are not dependent on the value of $P_{\mathrm{H}}$ in this power region.

The nondependence of $S D_{\mathrm{H}}$ on the value of thickness $t$ for the single bincho-charcoal plate is displayed in Fig. 7. In this figure, open squares, solid circles, open triangles, and solid diamonds represent the values of $S D_{\mathrm{H}}$ at $\mathrm{RF}$ frequencies of $1 \mathrm{MHz}, 10 \mathrm{MHz}$, $100 \mathrm{MHz}$, and $1 \mathrm{GHz}$, respectively, under a constant RF output power $P_{\mathrm{H}}$ of $33 \mathrm{dBm}$. It can be seen that the characteristics of $S D_{\mathrm{H}}$ for the single plate display no evidence of dependence on the values of $t$ in the region between $3 \mathrm{~mm}$ and $20 \mathrm{~mm}$. Namely, the $S D_{\mathrm{H}}$ is constant and not related to value of $t$ for the single plate in this thickness region. In addition, it was found that the $S D_{\mathrm{H}}$ as a function of $t$ for single plate displayed no dependence on the values of $P_{\mathrm{H}}$ in the region between $3 \mathrm{dBm}$ and $33 \mathrm{dBm}$. That is, the $S D_{\mathrm{H}}$ vs $t$ characteristics in this power region exhibited similar results for the frequency region from $1 \mathrm{MHz}$ to $1 \mathrm{GHz}$, such as

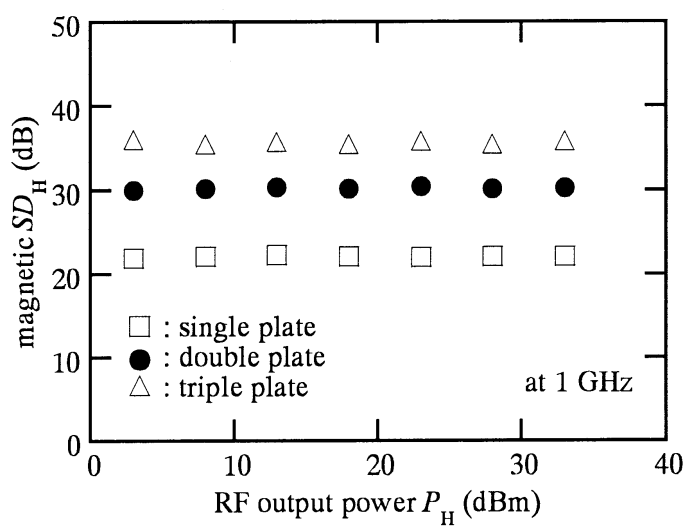

Fig. 6. Typical characteristics of $S D_{\mathrm{H}}$ for the bincho-charcoal plates at the frequency of $1 \mathrm{GHz}$ as functions of the RF output power $P_{\mathrm{H}}$. The open triangles, solid circles, and open squares represent the values of $S D_{\mathrm{H}}$ for the triple plate, double plate, and single plate, respectively.

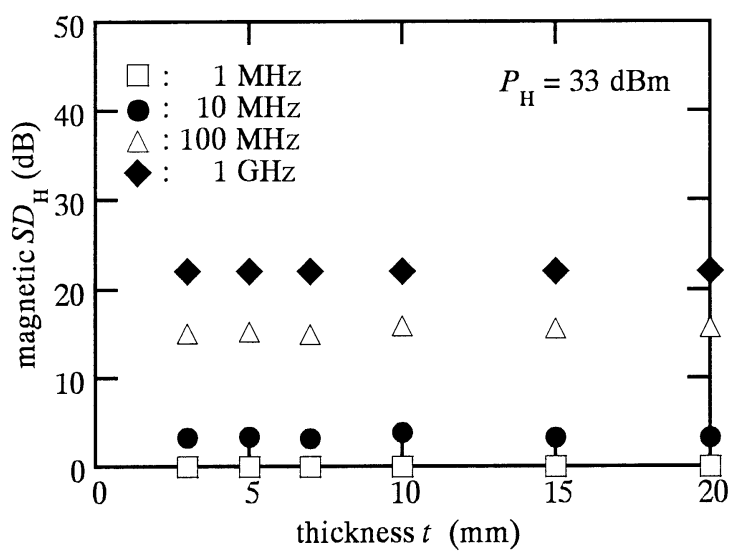

Fig. 7. RF magnetic shielding effect $S D_{\mathrm{H}}$ as a function of the thickness $t$ of the single bincho-charcoal plate. The open squares, solid circles, open triangles, and solid diamonds represent the values of $S D_{\mathrm{H}}$ at $\mathrm{RF}$ frequencies of $1 \mathrm{MHz}, 10$ $\mathrm{MHz}, 100 \mathrm{MHz}$, and $1 \mathrm{GHz}$, respectively, under a constant RF output power $P_{\mathrm{H}}(33 \mathrm{dBm})$ of the transmitting antenna. 


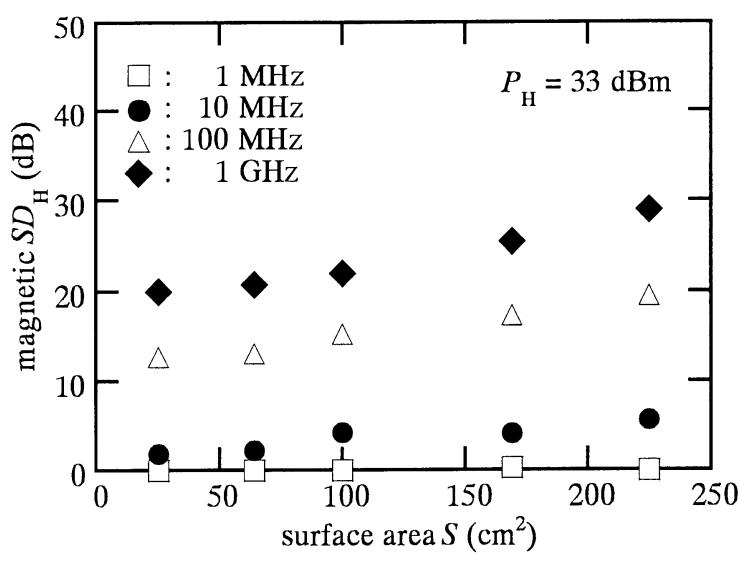

Fig. 8. Typical RF magnetic shielding degree $S D_{\mathrm{H}}$ as a function of the surface area $S$ of the single bincho-charcoal plate. The open squares, solid circles, open triangles, and solid diamonds represent the values of $S D_{\mathrm{H}}$ at $\mathrm{RF}$ frequencies of 1 $\mathrm{MHz}, 10 \mathrm{MHz}, 100 \mathrm{MHz}$, and $1 \mathrm{GHz}$, respectively.

shown in Fig. 7.

The plotted points in Fig. 8 denote the values of $S D_{\mathrm{H}}$ as a function of the surface area $S$ (25 225 cm square, $5 \mathrm{~mm}$ thick) of the single plate. In this figure, open squares, solid circles, open triangles, and solid diamonds represent the values of $S D_{\mathrm{H}}$ at $\mathrm{RF}$ frequencies of $1 \mathrm{MHz}, 10 \mathrm{MHz}, 100 \mathrm{MHz}$, and $1 \mathrm{GHz}$, respectively, under condition $\mathrm{RF}$ output power $P_{\mathrm{H}}$ of $33 \mathrm{dBm}$. It can be seen that the values of $S D_{\mathrm{H}}$ increase as the values of $S$ of the single plate increase in the frequency region from $10 \mathrm{MHz}$ to 1 $\mathrm{GHz}$. The values of $S D_{\mathrm{H}}$ at frequency $1 \mathrm{MHz}$, however, displayed little or no evidence of dependence on the surface area of the single plate in this surface region. Furthermore, the characteristics of $S D_{\mathrm{H}}$ such as shown in Fig. 8 displayed no dependence on the values of $P_{\mathrm{H}}$ in the region between $3 \mathrm{dBm}$ and $33 \mathrm{dBm}$.

\subsection{RF Electric Shielding Effect of Bincho-charcoal Plate}

The plotted points in Fig. 9 denote the RF electric shielding effect $S D_{\mathrm{E}}$ for the bincho-charcoal plates as functions of the RF frequency $f$, over the radio frequency range from $1 \mathrm{MHz}$ to $1 \mathrm{GHz}$, under a constant RF output power $P_{\mathrm{E}}(33 \mathrm{dBm})$ of the transmitting antenna. In this figure, the open squares, solid circles, and open triangles represent the values of $S D_{\mathrm{E}}$ for the single, double, and triple plates of charcoal, respectively. The RF electric shielding characteristics such as shown in this figure exhibit no remarkable effects. In addition, the characteristics of $S D_{\mathrm{E}}$ for the binchocharcoal plates displayed no evidence of dependence on the RF output power $P_{\mathrm{E}}$ in the range between $3 \mathrm{dBm}$ and $33 \mathrm{dBm}$ (not shown). The authors are presently investigating these characteristics in a theoretical analysis along with the experimental results.

3.4 Improvement of RF Magnetic Shielding Effect In order to further improve the RF magnetic shielding effect, a commercial copper plate (100 $\mathrm{mm}$ square, $1 \mathrm{~mm}$ thick) was superimposed over the triple charcoal plates, hereafter termed the superimposed plate. Figure 10 displays the typical RF magnetic shielding characteristics of three different plates as a function of frequency $f$, over the frequency range from $1 \mathrm{MHz}$ to $1 \mathrm{GHz}$, under a constant RF output power $P_{\mathrm{H}}(33 \mathrm{dBm})$. In this figure, the curves formed by the open triangles, open diamonds, and solid squares represent the shielding characteristics for the triple

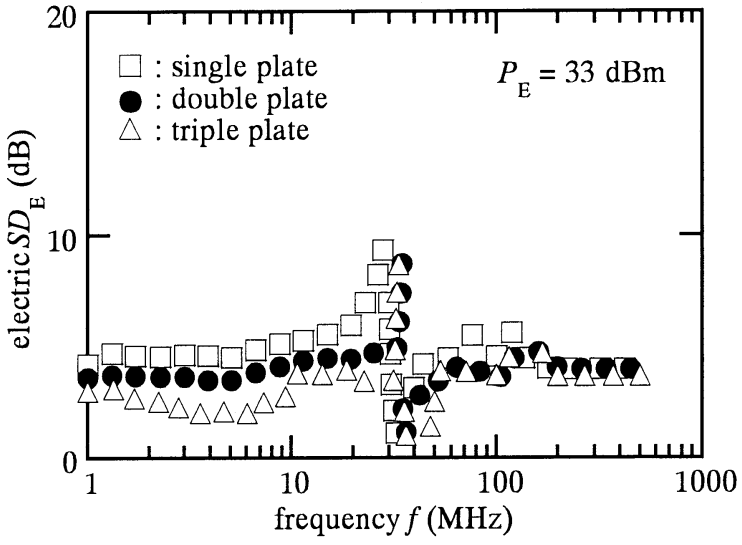

Fig. 9. Distributions of the RF electric shielding degree $S D_{\mathrm{E}}$ for the bincho-charcoal plates as functions of frequency $f$, under a constant RF output power $P_{\mathrm{E}}(33 \mathrm{dBm})$ of the transmitting antenna. The open triangles, solid circles, and open squares represent the values of $S D_{\mathrm{E}}$ for the triple plate, double plate, and single plate of bincho-charcoal, respectively.

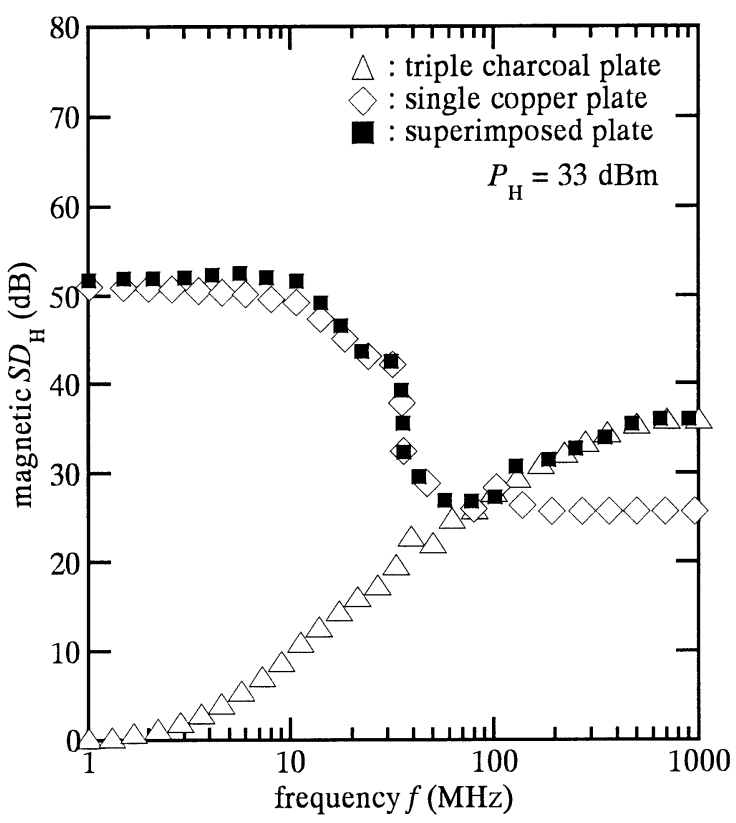

Fig. 10. Typical RF magnetic shielding characteristics of the various plates as functions of frequency $f$, under a constant RF output power $P_{\mathrm{H}}(33 \mathrm{dBm})$ of the transmitting antenna. The open triangles, open diamonds, and solid squares represent the values of $S D_{\mathrm{H}}$ for the triple bincho-charcoal plate, single copper plate, and superimposed plate, respectively.

bincho-charcoal, single copper, and superimposed plates, respectively. The values of $S D_{\mathrm{H}}$ are improved over that of the triple charcoal plate by superimposed the copper plate over the triple charcoal plate in the frequency region from $1 \mathrm{MHz}$ to 50 MHz. That is, the copper and triple charcoal plates are complementary to each other in the frequency region from $1 \mathrm{MHz}$ to $1 \mathrm{GHz}$, such as shown in Fig. 10. The present authors are now investigating the physical meaning of these characteristics. These results show an important criterion for improvement in the application of practical electromagnetic shielding. 


\section{Conclusions and Summary}

Previously, the electromagnetic characteristics of binchocharcoal as radio frequency (RF) shielding material had been unknown. Therefore, the present paper has examined and clarified the electromagnetic characteristics of bincho-charcoal, including the RF magnetic shielding degree $S D_{\mathrm{H}}$, the RF electric shielding degree $S D_{\mathrm{E}}$, and the relative permittivity $\varepsilon_{\mathrm{r}}\left(\varepsilon_{\mathrm{r}}{ }^{\prime}, \varepsilon_{\mathrm{T}}\right.$ "), in the RF region $(1 \mathrm{MHz}-1 \mathrm{GHz})$. Furthermore, it was shown how to improve the value of $S D_{\mathrm{H}}$ for the bincho-charcoal plates. The results of this paper can be summarized as follows:

(1) The characteristics of $\varepsilon_{\mathrm{r}}$ for the bincho-charcoal decreased as the frequency increased. The values of $\varepsilon_{\mathrm{r}}$ for bincho-charcoal are very large $\left(10^{7}-10^{2}\right)$ in the frequency region from $1 \mathrm{MHz}$ to 1 GHz, such as was shown in Fig. 4 (b).

(2) The value of $S D_{\mathrm{H}}$ for the triple plate at $100 \mathrm{MHz}$ was improved by about $15 \mathrm{~dB}$ over that of the single plate. In addition, the value of magnetic shielding degree $S D_{\mathrm{H}}$ was remarkably improved as a function of the superposition-layer number of the bincho-charcoal plates, such as was shown in Fig. 5.

(3) The values of $S D_{\mathrm{H}}$ are improved over that of the triple charcoal plate by superimposed the copper plate over the triple charcoal plate in the frequency region from $1 \mathrm{MHz}$ to $50 \mathrm{MHz}$. That is, the copper and triple charcoal plates are complementary to each other in the frequency region from $1 \mathrm{MHz}$ to $1 \mathrm{GHz}$, such as shown in Fig. 10.

These results represent important criteria fundamental in the design of practical RF shielding systems having greater reliability. The present authors are now investigating the physical meaning why values of $S D_{\mathrm{H}}$ are influenced by the air gap distance between the two bincho-charcoal plates in the double plate shield.

(Manuscript received Feb. 24, 2003, revised Jul. 4, 2003)

\section{References}

(1) Henry W. Ott : Noise Reduction Techniques in Electronic Systems, p. 1, John Wiley \& Sons, Inc., Canada (1988)

( 2 ) K. Itoh, Y. Sasai, Y. Hotta, and M. Itoh : "Improvement of RF Magnetic Shielding Effects of an HTS Cylinder: the Superposition of a Bincho-Charcoal Square Cylinder over a BPSCCO Cylinder", Physica C, Vol. 372-376, pp. 1333-1338 (2002)
( 3 ) Y. Hotta, K. Mori, and M. Itoh : Proc. 17th Int. Cryogenic Engineer. Conf., p. 507, Institute of Physic Publishing, Bristol and Philadelphia (1998)

( 4 ) M. Itoh, K. Itoh, K. Mori, and Y. Hotta : Applied Superconductivity 1999, p. 777, Institute of Physic Publishing, Bristol and Philadelphia (2000)

( 5 ) K. Itoh, Y. Hotta, M. Itoh, N. Munser, J. Plewa, W. Jaszczuk, and H. Altenburg : "RF Magnetic Shielding Effect of a Sealed Bottom HTS Cylinder", IEEE Trans. Appl. Superconductivity, Vol. 11, No. 1, pp. 2394-2397 (2001)

(6) S. A. Schelkunoff : Electromagnetic Waves, p. 306, Van Nostrand, Princeton (1943)

Keisuke Itoh (Member) graduated from the Department of Electronic Engineering of Kinki University,

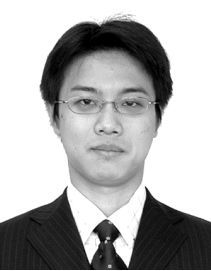
receiving a M. E. degree from Kinki University in 2001. He is presently a student in the doctoral course of the Interdisciplinary Graduate School of Science and Engineering, Graduate School of Kinki University, studying the RF magnetic shielding characteristics of an HTS plate. Mr. Itoh is a member of the Japan Society of Applied Physics and the Magnetic Society of Japan.

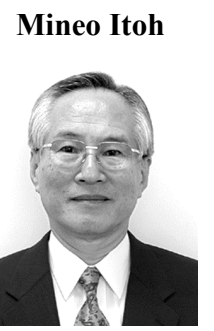

(Member) graduated from the Graduate School of Engineering of Kobe University, and received $\mathrm{M}$. E. and $\mathrm{Ph}$. D degrees in Engineering from Kobe University. Since 1966 he has been a member of the School of Science and Engineering, Kinki University, and currency holds the title of Professor. His research includes electronic measurements, the sensors made from HTS, the magnetic shielding using a superconductor, and the development of biosensors. Dr. Itoh is a member of the Japan Society of Applied Physics, the Society of Instrumentation and Control Engineering, and the Magnetic Society of Japan. 